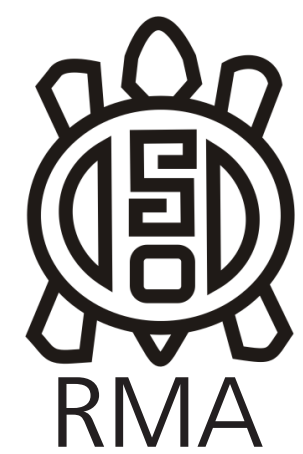

Arqueología

\title{
La cerámica y su rol social en contextos funerarios. PP9-III y PP13-I como casos de estudio (ca. 1000-1300 años ap) (Antofagasta de la Sierra, Catamarca)
}

\author{
Ceramics and their social role in mortuary contexts. PP9-III and \\ PP13-I as study cases (ca. 1000-1300 years bp) (Antofagasta de la \\ Sierra, Catamarca)
}

Vanesa Beatriz Juarez

Facultad de Ciencias Naturales e IML. Universidad Nacional de Tucumán, Argentina. E- mail: vanesajuarezb@gmail.com

\begin{abstract}
Resumen
Se presenta la información obtenida a partir de estudios estilísticos realizados en cerámicas recuperadas de contextos de inhumación correspondientes a los sitios Punta de la Peña 9-Sector 3 y Punta de la Peña 13-Sector I, localizados en la microrregión de Antofagasta de la Sierra (Puna Argentina). Así también, se efectúa un acercamiento al rol social de la cerámica en dichos espacios, dado que consideramos que sus características estilísticas junto con la historia de vida que portan los objetos son cualidades significativas en la selección de los mismos para incorporarlos a prácticas específicas como las funerarias. Con este fin se realizaron análisis sobre secciones delgadas de pastas cerámicas y se evaluaron los aspectos tecno-morfológicos, funcionales, decorativos y huellas de uso. Los resultados alcanzados permitieron abordar la variabilidad estilística presente en el conjunto cerámico estudiado y reconstruir la biografía de los objetos seleccionados para ser partícipes de las prácticas de inhumación.
\end{abstract}

Palabras Clave: Cerámica; Estilo; Historia de vida, Funebria, Puna.

\begin{abstract}
In this paper we present information obtained through stylistic analyses performed over ceramic materials recovered from mortuary contexts in the Punta de la Peña 9 -Sector 3-and Punta de la Peña 13-Sector I- sites. These sites are located in the Antofagasta de la Sierra micro-region (Argentinian Puna). Moreover, we seek to approach the social role of the ceramics in those spaces, since we consider that stylistic characteristics as well as the "life histories" of such objects, are meaningful qualities for the selection of these elements and their incorporation to specific practices such as mortuary ones. The analysis that allowed us to complete this study were performed on thin sections of ceramic pastes, observing techno-morphological, functional and decorative aspects, as well as wear traces. Finally, the achieved results helped us to keep expanding our knowledge of the stylistic variability present in the analyzed ceramic assemblage and to reconstruct the biography of the object chosen to participate in mortuary practices.
\end{abstract}

Keywords: Ceramic; Style; Life Histories; Mortuary Practices, Puna.

Según la información obtenida en más de 20 años de investigaciones sistemáticas llevadas a cabo en Antofagasta de la Sierra (ANS), los hallazgos de contextos funerarios, durante las ocupaciones cazadorasrecolectoras, comprendían la ubicación de los cuerpos directamente en abrigos rocosos u oquedades como es el caso del contexto que se ha conocido en la literatura como el "Bebé de la Peña" (Aschero et al. 2002; Aschero y Hocsman 2011). En relación al Período Formativo regional (sensu Olivera et al. 2006), se registraron depósitos funerarios con cuerpos de individuos humanos o partes de los mismos en estructuras construidas con rocas y emplastos de limo o arcilla (Babot et al. 2009; González
Baroni 2013; López Campeny 2009). Sin embargo, el registro de inhumaciones infantiles contenidas en vasijas y asociadas a fragmentos cerámicos nos muestra otra práctica mortuoria, hasta ahora no identificada en los primeros mil años de la era. Estos casos de estudio se localizaron en dos sitios particulares: Punta de la Peña 9- Sector 3 y Punta de la Peña 13- Sector I (en adelante PP9-III y PP13-I) (Figura 1). A partir del reconocimiento de esta práctica funeraria, utilizando recipientes cerámicos, nos preguntamos sobre las razones que habrían influido en la selección de esos contenedores para llevar a cabo estas inhumaciones en ANS.

En este trabajo, nos interesamos en abordar las prácticas 


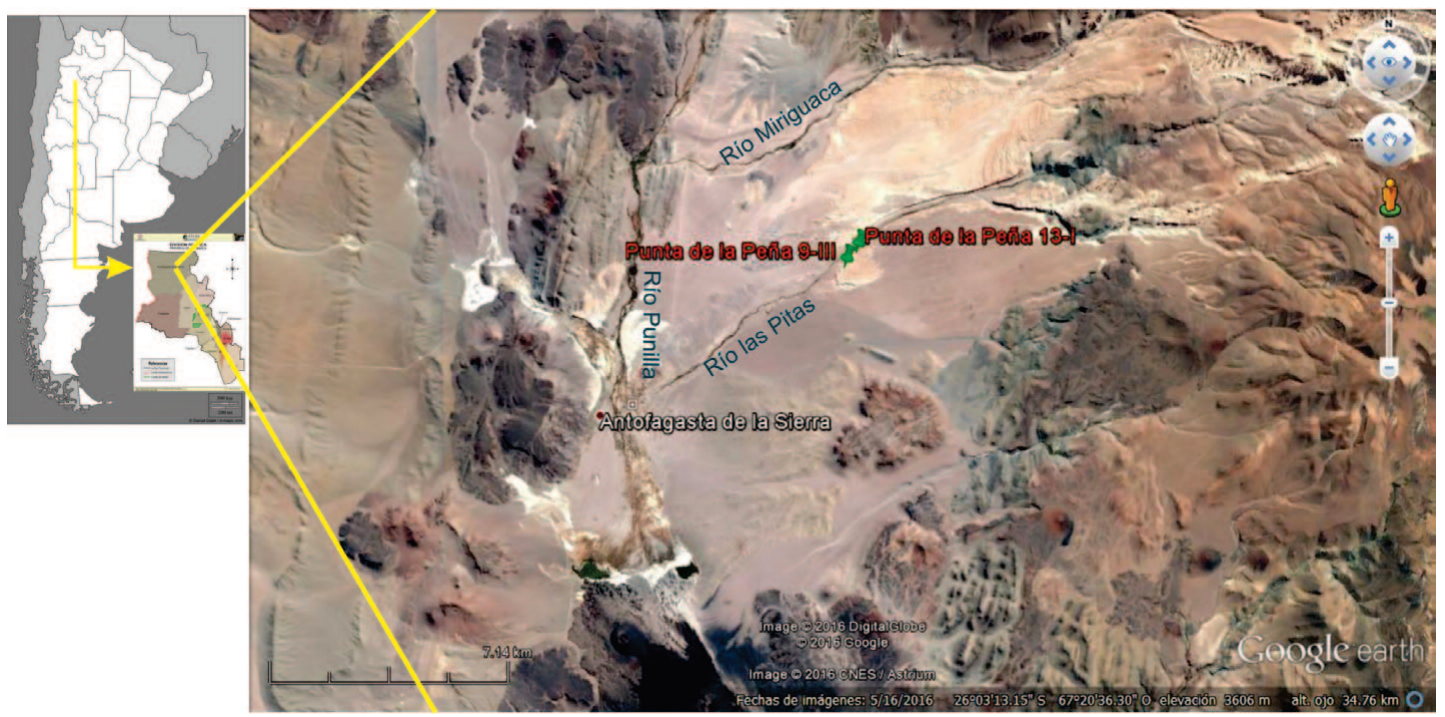

Figura 1. Imagen satelital extraída de Google Earth. Sitios PP9-III y PP13-I.

Figure 1. Satellite image taken from Google Earth. Sites PP9-III and PP13-I.

sociales relacionadas al material cerámico en toda su trayectoria de vida, por lo cual nos interrogamos acerca de los aspectos estilísticos representados, el estado de integridad y las formas de uso que primaron en la selección de los mismos para su incorporación en los contextos de inhumación (Gosden y Marshall 1999).

Para estudiar los aspectos estilísticos de la cerámica se tuvo en cuenta la suma de atributos que componen a una pieza, siendo éstos los tecnológicos, morfológicos, funcionales y decorativos, que en conjunto nos otorgan un panorama de las selecciones realizadas por el artesano de entre un abanico de posibilidades que corresponden a un espacio y tiempo determinados (Hodder 1990). Por otra parte, en relación a la integridad de la cerámica se analizó el estado de conservación en que ésta fue hallada, como así también se estudiaron los rastros de usos que permitan asociar estos artefactos a actividades previas a las funerarias y que pudieron afectarlas físicamente (Skibo 1992). Para poder abordar los objetivos planteados, se realizaron análisis relacionados con la identificación de las materias primas utilizadas en la manufactura cerámica; la caracterización de las representaciones estilísticas; como así también la evaluación de las huellas y formas de uso de los artefactos en los contextos pre-funerarios y funerarios.

\section{Antecedentes}

Teniendo en cuenta que en este trabajo nos interesamos por las prácticas de inhumación en contenedores cerámicos registradas en ANS, pasamos a mencionar el único registro conocido hasta el momento en la microregión que muestra ciertas semejanzas, en algunos aspectos, a los casos abordados por nosotros en los sitios PP9-III y PP13-I. Se trata del sitio Piedra Horadada 2 (PH2). La inhumación fue datada en $1270 \pm 50$ años AP y fue relevada en un alero o estructura de reparo bajo roca (nivel 1). De allí se recuperó un fragmento de corona de esmalte dental humano asociado a un conjunto de fragmentos cerámicos que remontados permitieron identificar el perfil globular de una pieza, de cuello recto evertido, con decoración mediante técnica de pastillaje en el sector del borde y asas a la altura del diámetro máximo del cuerpo. Este contenedor fue interpretado como una posible urna, la cual se asemeja en forma y decoración a uno de los recipientes recuperados en PP13-I (López Campeny 2009). Así también, se halló próxima a la corona de esmalte dental, una escudilla fragmentada cuyo borde presentaba un decorado con representaciones geométricas mediante incisiones. Cabe mencionar además, que en la misma estructura, pero en el nivel 3, se recuperó un fragmento de vértebra lumbar correspondiente a un individuo de 2 o 3 meses de edad (López Campeny 2009).

Considerando la escasez de antecedentes respecto a la utilización de contenedores cerámicos para la depositación de restos óseos humanos, indagamos en aquellos trabajos que pudieran aportar datos sobre las prácticas de inhumación registradas en ANS. De esta manera, observamos que los entierros relevados para el Formativo regional, poseen en general, características diferentes a las inhumaciones de individuos en recipientes cerámicos.

Los depósitos funerarios frecuentemente registrados en ANS consisten en conjuntos de restos óseos desarticulados e incompletos pertenecientes a uno o más individuos. Estas acumulaciones óseas resultaban de la extracción y reubicación de algunas partes como práctica mortuoria de exhumación (Martínez y Aschero 2005). Los restos mencionados se encontraban inmersos en estructuras delimitadas por rocas con emplasto rojo (de silicatos en 
tamaño arcilla-limo) y acompañandos por fragmentos cerámicos semejantes a la cerámica Ciénaga y Saujil; artefactos líticos; y restos vegetales locales y alóctonos como endocarpos de chañar (Geoffroea decorticans), de algarrobo negro y blanco (Prosopis nigra y $P$. alba) y marlos de maíz (Zea mays) (Babot et al. 2009; López Campeny 2009).

A raíz de la revisión de los antecedentes apreciamos que, aunque las inhumaciones de ANS en general no utilizaron contenedores cerámicos, existen importantes similitudes entre los contextos funerarios locales y los de PP9-III y PP13-I. Entre los elementos compartidos podemos mencionar, por ejemplo la utilización de un pigmento o emplasto rojizo tanto para la preparación de un piso como para la unión de rocas que constituyen una cista. Así también esta aplicación se usó para el engalanado de los componentes del contexto funerario y/o la aplicación post-cocción en cerámica en forma de manchas (Krapovickas 1955). El acondicionamiento mediante la aplicación de una pigmentación mineral rojiza, parecería conformar parte de una costumbre puneña frecuentemente registrada en los hallazgos funerarios de ANS y otras zonas como Tebenquiche Chico y Puna de Atacama (Babot et al. 2009, González Baroni 2013, Krapovickas 1955, entre otros).

Por otra parte, observamos una recurrencia en el resguardo de partes esqueletales de uno o más individuos, como práctica funeraria local con una antigüedad de casi 10.000 años. Estas deposicitaciones de partes esqueletales de los individuos, fueron interpretadas como símbolos identitarios y de demarcación territorial (Aschero 2007). La dinámica evidenciada en la mayoría de los casos mencionados de inhumación y exhumación pueden tener una estrecha relación con una concepción de los ancestros como medios para reclamar el acceso o desplazamiento por el territorio de ANS y acceder a los recursos (Aschero 2007).

Como se puede apreciar, las inhumaciones registradas hasta el momento en ANS no se caracterizan por efectuarse dentro de contenedores cerámicos. Sin embargo, éstas prácticas fueron relevadas en el área valliserrana y tierras bajas orientales del Noroeste argentino, para el primer milenio de la era. Del abanico de antecedentes que estas áreas ofrecen, nos interesamos particularmente por el uso de contenedores para inhumación localizados en regiones inmediatamente adyacentes a ANS como el Valle de Hualfín (Provincia de Catamarca). Esta restricción espacial se realiza teniendo en cuenta que la cerámica valliserrana (Ciénaga y Saujil) incrementa de manera notable su representatividad en ANS, con posterioridad a los 2000 años AP (López Campeny 2012; Olivera 1992; Olivera et al. 2006).

Finalmente, considerando la semejanza morfológica y decorativa propuesta por López Campeny et al. (2014)

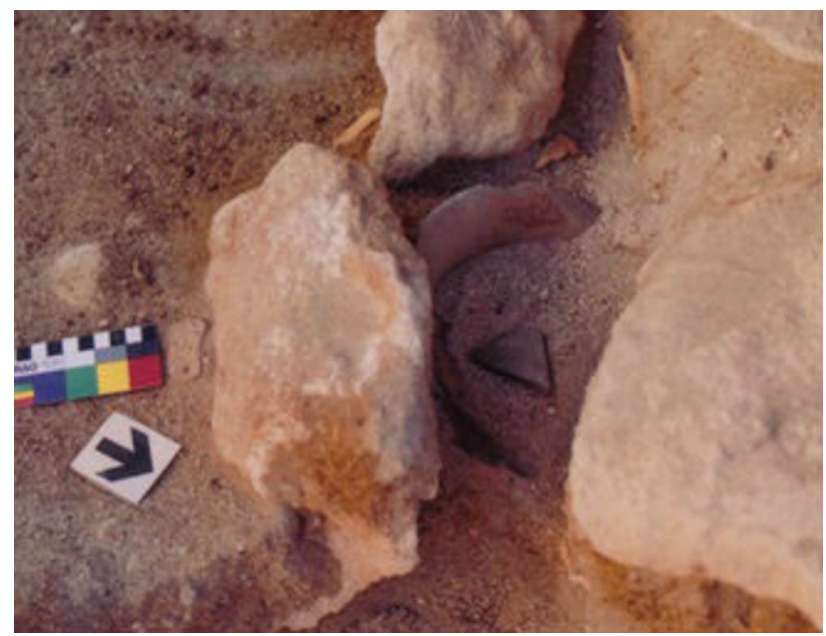

Figura 2. Contexto funerario de PP9-III. Foto cortesía de Cohen (2005)

Figure 2. Funerary context of PP9-III. Photo courtesy of Cohen (2005).

entre algunas cerámicas del contexto de inhumación de PP13-I y la alfarería registrada en el sector meridional de las selvas occidentales, profundizaremos el análisis estilístico comparativo entre los recipientes recuperados en ANS y los relevados en contextos funerarios de las tierras bajas orientales.

\section{PP9-III y PP13-I como casos de estudio}

El sitio PP9-III (coordenadas geográficas 26 $01.631^{\prime}$ Latitud S y $67^{\circ} 20.498^{\prime}$ Longitud O/ 3620 msnm), contiene al contexto de inhumación localizado en lo que se denomina Estructuras 3+4, a una profundidad de $60 \mathrm{~cm}$, en la Capa 6, que fue relacionada con una ocupación de corral (1290 \pm 50 años AP (UGA 9261)) (Cohen 2005,2010). Aquí se registró un conjunto óseo incompleto correspondiente a un neonato de 4 meses

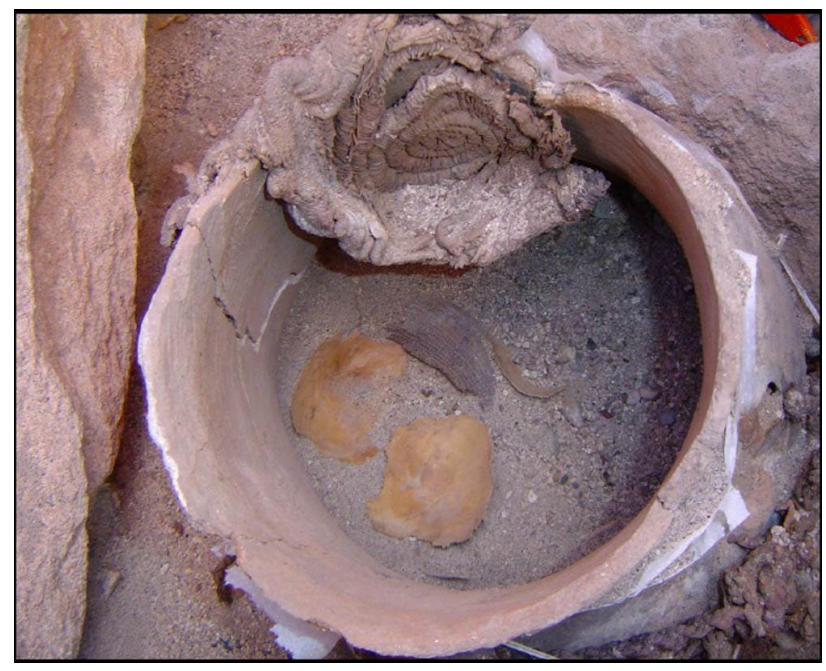

Figura 3. Contexto funerario A de PP113-I. Foto cortesía de Martel.

Figure 3. Funerary context A of PP13-I. Photo courtesy of Martel. 
de edad aproximadamente, datado en $1090+50$ años AP (UGA 15106), el cual se encontraba inmerso en el interior de un contenedor cerámico (en adelante Pieza 1) (Figura 2). Este recipiente se presentaba muy deteriorado, fragmentado y conservaba el $50 \%$ de su cuerpo, ausentándose la base. Asociada a la inhumación se registró un ajuar conformado por instrumentos líticos completos y fragmentados, desechos de talla, un núcleo, un fragmento de cuenta de malaquita, restos vegetales locales y alóctonos, y un fragmento cerámico con incisiones. El contexto integral que implica una inhumación en un corral fue interpretado como un acto de ofrenda de un individuo humano neonato vinculado con la prosperidad de la hacienda o la suerte (Cohen 2010).

En relación al sitio PP13-I (coordenadas geográficas $26^{\circ}$ 0' 9.51 "Latitud S y 67 20'27.6" Longitud O/ 3602 $\mathrm{msnm})$, se registró aquí un gran bloque ignimbrítico con representaciones rupestres del Período Tardío o Inka. Al pié de este se halló una estructura funeraria subterránea (cista) en donde se relevaron dos inhumaciones. La primera constituye el Contexto Funerario A, el cual está conformado por un contenedor cerámico (en adelante Pieza 2) envuelto en una red de fibra vegetal, datada en $1280 \pm 60$ años AP (LATYR-1723) (López Campeny 2009; Martel 2005). En el interior del recipiente cerámico se depositaron los restos óseos, articulados con tejido blando, de un neonato de 2 meses de edad aproximadamente, junto con 2 cestas elaboradas en fibra vegetal y 90 cuentas en mineral identificado como turquesa (Figura 3). En superficie se recuperó un puco gris fragmentado (en adelante Pieza 3) (López Campeny 2009; Martel 2005).

Por otra parte, la segunda inhumación que conforma al Contexto Funerario B, constituye un entierro secundario y se encuentra fuera del contenedor cerámico envuelto en fibra vegetal (Pieza 2), pero aún dentro de los límites de la cista (Del Bel et al. 2006). En este lugar se registró un tercer depósito funerario con un conjunto incompleto de restos óseos de neonato asociado a un fragmento cerámico con incisiones (en adelante Fragmento 1), recuperado de las arenas que rellenaban a la estructura funeraria (Martel 2005). Los contextos de inhumación fueron diferenciados como A y B por encontrarse en diferentes posiciones respecto de la Pieza 2 y por diferencias en las prácticas funerarias asociadas. Sin embargo se desconoce aún si los contextos son sincrónicos o diacrónicos ya que no se realizaron dataciones directamente sobre los restos óseos depositados en ellos sino sobre los restos vegetales asociados.

\section{Metodología}

El conjunto cerámico estudiado está constituido por tres piezas incompletas y cuatro fragmentos cerámicos. Por un lado, una pieza incompleta procedente del sitio PP9III (Pieza 1), mientras que el fragmento con incisiones asociado al mismo contexto se encuentra siendo analizado por otra investigadora en la Universidad Nacional de La Plata. Por otra parte, dos piezas incompletas (Pieza 2 y 3) y cuatro fragmentos remontables (Fragmento 1) se recuperaron en PP13-I. Para aproximarnos a conocer los estilos presentes en la cerámica, nuestro análisis se enfocó en las características tecno-morfológicas, funcionales y decorativas. Para ello nos guiamos por los lineamientos metodológicos establecidos en la Primera Convención Nacional de Antropología (1966), Rice (1989), Balfet et al. (1992), Orton et al. (1997) y Wynveldt (2006). Este abordaje hizo posible, además, acercarnos a dilucidar parte de la historia de vida de los objetos cerámicos y particularmente a su relación con las prácticas sociales de inhumación. Por este motivo se analizaron también las huellas de uso (sensu Skibo 1992).

Se llevaron a cabo los siguientes estudios:

Caracterización petrográfica de pastas en secciones delgadas con registro en fichas estandarizadas confeccionadas por Balesta y Zagorodny para el Laboratorio de Análisis cerámico de la Facultad de Ciencias Naturales y Museo de la Universidad Nacional de La Plata. En dichas fichas se registraron las siguientes características: textura y color de la matriz arcillosa; orientación, densidad y forma de las cavidades; densidad, esfericidad, orientación, tamaño y naturaleza de los antiplásticos, los cuales fueron considerados como aquellas inclusiones no plásticas superiores a $15 \mu \mathrm{m}$.

Por otra parte, se tuvo en cuenta tipo de cocción de la cerámica, tratamiento de superficie, parte de la pieza a la que pertenece el fragmento, espesor de paredes y forma de la pieza. La caracterización de cada ítem se efectuó teniendo en cuenta los conceptos postulados en la Primera Convención Nacional de Antropología (1966).

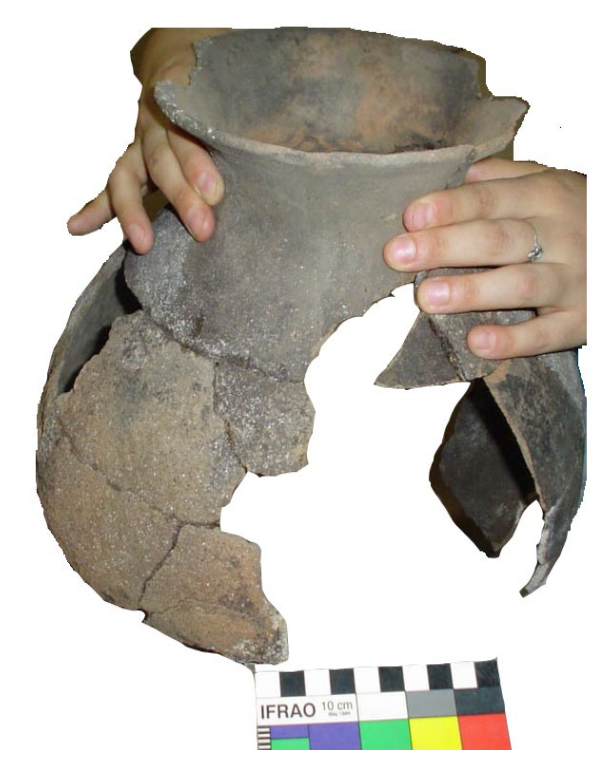

Figura 4. Pieza 1. PP9-III. Foto cortesía de Cohen (2005).

Figure 4. Piece 1. PP9-III. Photo courtesy of Cohen (2005). 
Estudios decorativos: Este aspecto implicó el registro de la aplicación de pintura; aplicación de pastillaje, incisiones y grabados. También se tuvieron en cuenta los motivos o diseños representados en las piezas/ fragmentos y su disposición. La técnica decorativa se determinó según lo postulado por la Primera Convención Nacional de Antropología (1966).

Estudios funcionales: Analizar esta propiedad implicó el registro de característica tales como forma de la pieza, estabilidad, capacidad de volumen, espesor de las paredes, accesibilidad (Rice 1989).

Estudios de Huellas de Uso: Se registró el estado de conservación en que se encontraron piezas y fragmentos y se trató de identificar alteraciones antrópicas en las propiedades de los artefactos como resultado del uso de los mismos. Se distinguieron dos clases de alteraciones según la propuesta de Skibo (1992): adhesión de material y remoción de material. La búsqueda de rastros de uso se realizó a nivel macroscópico y sub-macroscópico (con lupa digital portátil de 25x a 400x) tanto en la superficie externa como interna de los fragmentos cerámicos.

Finalmente, también para obtener indicios de usos preinhumación en las Piezas 1 y 2, se realizaron análisis de micro-fósiles de muestras obtenidas mediante raspados en húmedo en el interior de las mismas ${ }^{1}$. Este procedimiento se realizó en el sector del diámetro máximo de las piezas. El análisis de muestras se llevó a cabo con un microscopio de polarización (Zeiss Aixioskop), y fue de índole cualitativo para identificar diversos microfósiles presentes en las muestras. Para la identificación de morfotipos se utilizó la colección de referencia de plantas útiles, del laboratorio del Instituto de Arqueología y Museo (Facultad de Ciencias Naturales e Instituto Miguel Lillo. UNT).

\section{Resultados: Representaciones estilísticas y biografía de la cerámica}

\section{PP9-III: Pieza 1.}

Sobre las propiedades relevadas en este recipiente podemos decir que el mismo consiste en un contenedor de forma sub-globular y de mediano a gran tamaño (30 cm de alto) que permite contener hasta 17 Lts. Posee tratamientos de superficie de alisado y peinado localizados en diferentes sectores. Su pasta es de cocción oxidante, porosa, poco compacta y presenta inclusiones de cuarzos de tamaños variables, algunas distinguibles a ojo desnudo (Figura 4).

Respecto a las huellas de uso, se identificaron rastros relacionados al procesamiento de sustancias y exposición al fuego de la pieza. Este reconocimiento se realizó

\footnotetext{
${ }^{1}$ Estas tareas las llevó a cabo la Técnica Asistente Julieta Zapatiel del Laboratorio de Arqueobotánica del Instituto de Arqueología y Museo - ISES CONICET. Facultad de Ciencias Naturales e IML, UNT.
}

mediante la observación de desgastes por incisiones y raspados localizados en el interior del contenedor, como así también la presencia de depósitos de hollín en la superficie externa y de micro-carbones. Asimismo, se identificaron, en forma aislada y en cantidades poco significativas, fragmentos de tejidos vegetal deshidratado, silicofitolitos y calcifitolitos. La predominancia de microcarbones registrados se considera relevante y permiten asociar la pieza al procesamiento-cocción de alimentos (Haros 2014). Desde lo funcional también identificamos propiedades de la pieza (como forma, capacidad de volumen, accesibilidad y espesor de las paredes) que pudieron acondicionarla para intervenir de forma eficaz en actividades domésticas y particularmente las relacionadas con el procesamiento de recursos como pudo ser la cocción de alimentos.

En relación a la pasta podemos decir que ésta posee una compactación baja. La matriz arcillosa presenta un color castaño oscuro y textura grumelosa. Sus cavidades poseen formas lenticulares y redondeadas, se presentan en porcentajes bajos ( $<5 \%)$ y orientadas en forma regular parcial. Como parte de la matriz arcillosa se identificaron muscovitas aciculares y cuarzos de angulares a subangulares. Como antiplásticos ser registraron cuarzos monocristalinos, plagioclasas, muscovitas, biotitas, feldespatos potásicos, y fragmentos de granito. Estas inclusiones son angulares y subangulares, y se presentan en porcentajes del 20 al 30\% sin orientación. Así también, los clastos poseen granulometría variables, de limo media a arena gruesa.

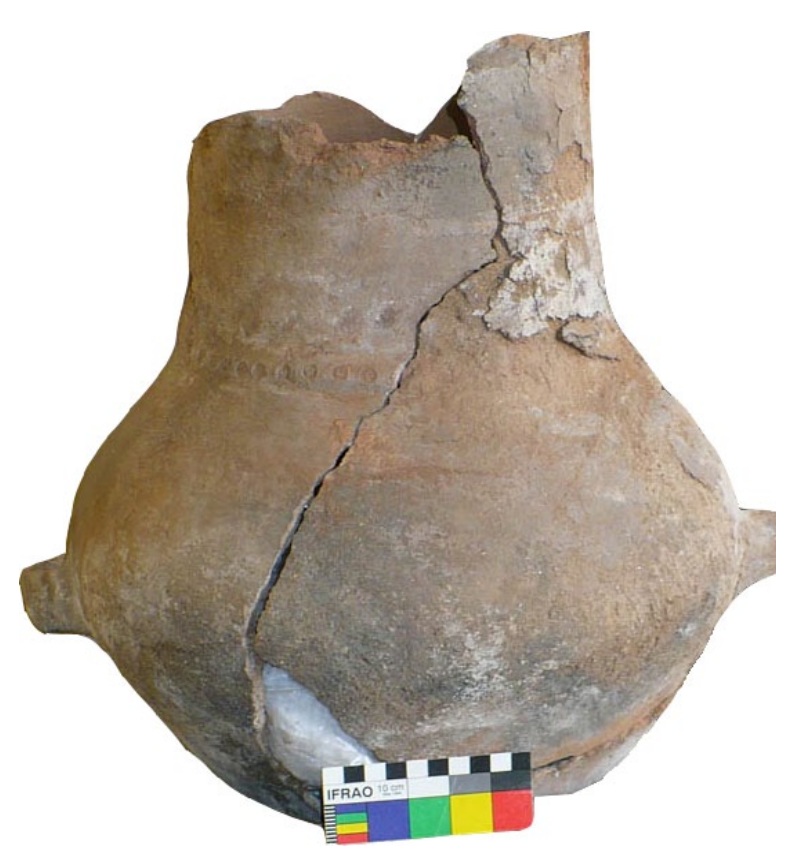

Figura 5. Pieza 2. PP13-I. Foto cortesía de Martel (2011).

Figure 5. Piece 2. PP13-I. Photo courtesy of Martel (2011). 
Teniendo en cuenta las características petrográficas de la Pieza 1, como la presencia de huellas de uso relacionados con la exposición al fuego, y los atributos morfológicos y funcionales registrados, consideramos que existe una posible correspondencia con lo que López Campeny (2012) y Puente (2015) identifican como cerámica local en ANS para antes y después del 1000 d.C. Sin embargo, sobre el componente plutónico de la pasta, la bibliografía geológica consultada nos menciona que las fuentes más próximas a los contextos analizados, en la región puneña, se encuentran en el Cerro Tebenquicho y Cerro Archibarca ubicados al Norte de Antofalla, pues geológicamente el área es predominantemente volcánico (González 1983, Palma et al. 1986). Asimismo, estos recursos son factibles de hallar a nivel extra-regional como sierras, valles y tierras bajas de la vertiente oriental (Mapa geológico de Catamarca 1995; Mapa geológico de Jujuy 1996; Mapa geológico de Salta 1998; Mapa geológico de Tucumán 1994). Por tal motivo consideramos de fundamental importancia profundizar a futuro en los estudios de procedencia.

Por su parte, como se mencionó anteriormente, las fuentes graníticas puneñas más cercanas se localizan a una distancia aproximada de $80 \mathrm{Km}$ respecto de los hallazgos en PP9-III. Desde esta perspectiva y teniendo en cuenta que para Arnold (2005) los materiales locales se encuentran en un radio de 7 a $10 \mathrm{~km}$, proponemos que la manufactura de la Pieza 1 implicó el uso de recursos no locales.

\section{PP13-I: Contexto Funerario A}

Pieza2

La integración de las características tecno-morfológicas, decorativas y funcionales hizo posible reconocer en la Pieza 2 una cerámica poco compacta, de cocción oxidante, con aplicación de engobe y alisado como parte del tratamiento de superficie Su decoración de destaca por la aplicación de pastillaje en el que se estamparon círculos de forma rítmica (Figura 5). La asociación entre estas propiedades, la forma de la pieza (de cuerpo globular y cuello recto-evertido) y el uso funerario asignado a la misma nos permite dilucidar semejanzas entre este recipiente y las urnas de estilo Candelaria descriptas por Heredia (1974) en el sector meridional de las selvas occidentales del NOA. Sobre éstas últimas, el autor menciona que desde los 400 d.C, los perfiles compuestos de piezas con cuerpo globular o sub-globular, base convexa y cuello recto, constituyen características recurrentes en urnas Candelaria. Teniendo en cuenta esta caracterización, pensamos que estas propiedades se hicieron evidentes en la Pieza 2, dado que la misma posee tanto la forma como una capacidad de volumen que permite resguardar los restos óseos de un neonato de aproximadamente 2 meses de edad².
Debido a la ausencia de huellas de uso previas a la inhumación, consideramos que este contenedor cerámico habría sido creado exclusivamente con fines funerarios, es decir con función de urna. Si bien se identificaron microfósiles de micro-carbones, silicofitolitos, cristales de calcio y diatomeas en el sector interno del recipiente, éstos no se hallaron en cantidades diagnósticas como para asignar funcionalidades o usos particulares a la pieza. Tomando en consideración este posible uso exclusivamente funerario de la Pieza 2, se tuvo en cuenta también la presencia de pequeños orificios de $2 \times 2 \mathrm{~cm}$, localizados en el cuerpo y en la base. Interpretamos que estos agujeros pudieron resultar de prácticas de "matado", las cuales consisten en el rompimiento intencional de objetos como parte de un rito fúnebre (Ambrosetti 1906, Román Marambio y Cantarutti Rebolledo 1998). Compartimos esta interpretación sobre todo porque el contexto de PP13-I corresponde a un espacio funerario. Sin embargo, es llamativo que los agujeros mencionados se encuentren cubiertos por una pasta arcillosa sin cocción. A partir de esto, inferimos la participación de la pieza en dos tradiciones o prácticas funerarias representativas de dos regiones distintas. Sobre estas condiciones del recipiente volveremos más adelante, cuando proponemos una interpretación sobre la procedencia del contenedor.

En relación a la caracterización de la pasta, el abordaje petrográfico realizado en este trabajo evidenció una cerámica cuya matriz arcillosa presenta color castaño oscuro y textura pseudolepidoblástica. Sus cavidades poseen formas lenticulaes, se presentan en porcentajes moderados (5-15\%) y con orientación regular parcial. Como parte de la matriz arcillosa se identificaron finas laminillas de biotita e inclusiones muy finas de cuarzo. Como antiplásticos se registraron cristaloclastos de cuarzo policristalino, feldespato potásico, biotita, plagioclasa y hornblenda. Así también, se identificaron litoclastos de granito. Estas inclusiones son angulares y subangulares con una orientación regular parcial, y están representadas en porcentajes de entre el 20 y 30 \%. Los clastos poseen granulometría de limo medio a arena media.
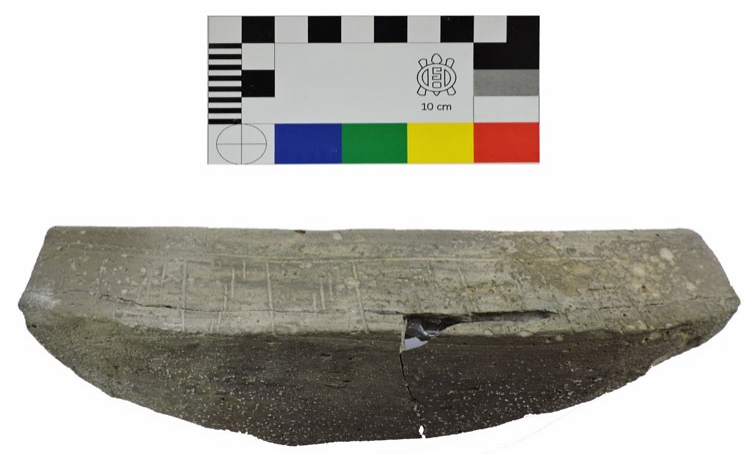

Figura 6. Pieza 3. PP13-I.

Figure 6. Piece 3. PP13-I. 
Los componentes de la pasta pueden interpretarse como pertenecientes a ambientes plutónicos, al igual que la Pieza 1. Asimismo, esos recursos se encuentran a distancias considerables por lo que son considerados como no locales. En relación a esto, para abordar la interpretación relacionada con la posible procedencia de la Pieza 2, los datos anteriores serán complementados con información correspondiente a otros elementos que forman parte del mismo contexto funerario.

En relación a los materiales y prácticas asociadas a la Pieza 2 , tuvimos en cuenta la forma en que se encontraron los restos del neonato contenido en el recipiente cerámico. El mismo se conservaba íntegro, sin presentar faltantes que pudieran dar indicios de algún proceso de remoción y traslado de ciertas partes óseas como ocurre con las prácticas funerarias locales más recurrentemente registradas en ANS. Por otra parte, el contenedor del individuo se encontraba asociado, en el Contexto Funerario A, a un puco o escudilla, localizado próximo a la boca de la Pieza 2, que podría haberse utilizado como tapa de la misma, como sucedió con las urnas del sector meridional de las selvas occidentales (Heredia 1974; López Campeny et al. 2014). Esta asociación entre urnas y tapas, en relación al estilo Candelaria de la Pieza 2, y al tratamiento mortuorio del neonato que no involucró el traslado de ciertas partes esqueletales, nos permite poner en consideración que las características del Contexto Funerario A pueden estar representando prácticas funerarias foráneas a la micro-región de ANS y semejantes a las de las poblaciones de las tierras bajas orientales.

Por otra parte, se interpreta que los orificios localizados en el cuerpo y base del contenedor corresponden a prácticas de matado, como sucedió con otros recipientes registrados por Ambrosetti en Pampa Grande (Salta) (Ambrosetti 1906). De esta manera creemos que la pieza participó de dos tradiciones funerarias que responden a poblaciones diferentes. A modo hipotético podemos proponer, por un lado, que la pieza pudo ser manufacturada en las tierras bajas orientales, con la finalidad de utilizarla como recipiente funerario con los tratamientos (de matado) que ello implica. Por otro lado, teniendo el posterior encubrimiento de los orificios, consideramos que éste indicaría una posible intervención por parte de personas puneñas, pues el acondicionamiento de espacios funerarios y ajuares, mediante la utilización de emplastos rojizos, es una práctica frecuentemente registrada en ANS (Babot et al. 2009; Krapovickas 1955; López Campeny 2009; Martínez et al. 2010).

Así también, la identificación de especies vegetales presentes en el contexto permitió efectuar algunos acercamientos a la procedencia de la Pieza 2. Sobre los artefactos confeccionados en fibra vegetal, se detectaron algunas especies de amplia distribución regional y otras diagnósticas de origen alóctono, (Acrocomia chunta; del género Bromelia, las especies $B$. hyeronymi Mez; $B$. serra Griseb; B. urbaniana (Mez)) y típicas de áreas ecológicas muy húmedas y distantes de ANS como por ejemplo Salta, Tucumán, Chaco, Formosa y Jujuy. Esta información se complementó con el reconocimiento de taxones de polen, extraídos del interior de la Pieza 2, entre los que no se registraron ejemplares de familias polínicas locales como Verbenaceae, Asteraceae, Cyperaceae, las cuales están presentes en todos los perfiles sedimentarios de ANS y en la lluvia polínica actual (López Campeny et al. 2014). Pieza 3

Esta pieza corresponde a un contenedor de pequeño o mediano tamaño como una escudilla de perfil compuesto, que posee una pasta gris, compacta, de cocción reductora y tratamiento se superficie de alisado junto con una decoración de grabado de líneas paralelas dispuestas escalonadamente (Figura 6). Objetos como éstos fueron registrados por Heredia (1974) en el sector sur de las selvas occidentales del Noroeste Argentino, para momentos posteriores al 400 d.C, como característicos de la Fase Choromoro de Candelaria. Asimismo, el grabado presente en la Pieza 3 se asemeja a la decoración registrada en la cerámica de la Fase Chuscha de Candelaria (sensu Heredia 1974). Para el 1000 d.C las escudillas son también frecuentemente registradas siendo utilizadas como tapas de contenedores funerarios, a la vez que también se utilizaron en otras ocasiones, fragmentos cerámicos de otras vasijas (Heredia 1974). Estas formas de uso en contextos funerarios también fueron registradas en sitios del Norte de Tucumán (Berberián 1969).

En relación a la participación de estos objetos en otros espacios y actividades, cabe mencionar la ausencia de huellas de uso de naturaleza antrópica, que permitan asociar a este artefacto con contextos distintos de los funerarios. En cambio, especialmente en la superficie externa, se identificaron alteraciones en el tratamiento de superficie que interpretamos como resultado de
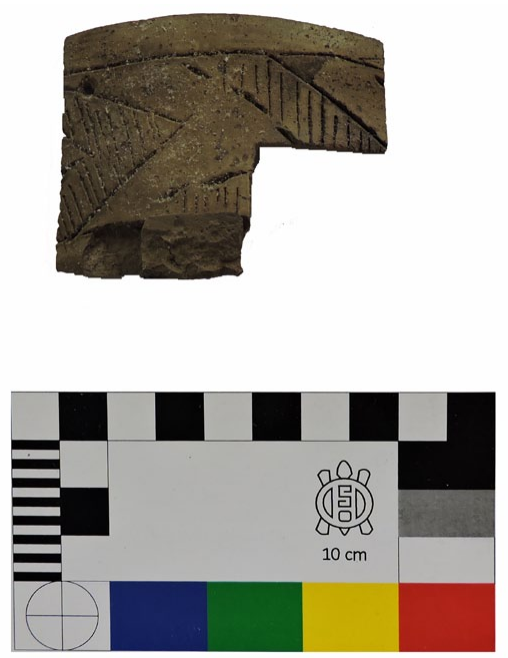

Figura 7. Fragmento 1. PP13-I.

Figure 7. Fragment 1. PP13-I. 
la erosión natural la cual provocó hoyos o piquetes, también llamados efecto pedestal (sensu Skibo 1992). Desde este punto de vista, teniendo en cuenta las características representadas en el contenedor y el espacio de inhumación del que forma parte en PP13-I (en asociación a la Pieza 2), creemos que es posible asociar la Pieza 3 al estilo cerámico Candelaria de las tierras bajas occidentales.

En relación a los recursos seleccionados para la manufactura de la Pieza 3, identificamos que su pasta compacta se caracteriza por poseer una matriz arcillosa de color castaño oscuro y textura pseudolepidoblástica. Sus cavidades se presentan en porcentajes bajos $(<5 \%)$, con forma y orientación irregular. Como parte de la matriz arcillosa se identificaron finas laminillas de biotita e inclusiones muy finas de cuarzo. Los antiplásticos registrados fueron cristaloclastos de cuarzo monocristalino y policristalino, feldespato potásico, mica, anfíboles y plagioclasas. Así también, se identificaron litoclastos de andesita y material piroclástico como pómez. Estas inclusiones son subangulares y subredondeadas con una orientación irregular, y están representadas en porcentajes de entre el 20 y 30 \%. Los clastos poseen granulometría de limo medio a arena media. Teniendo en cuenta especialmente la presencia de componentes volcánicos, como andesita y material piroclástico, consideramos que la cerámica pudo ser elaborada localmente. La manufactura pudo implicar el uso de los recursos más representativos que éste lugar provee, dado que se trata de una región con componentes geológicos primordialmente volcánicos, pero representando estilísticamente a una cerámica alóctona como Candelaria.

\section{Contexto Funerario B de PP13-I}

\section{Fragmento 1:}

Este fragmento representa una cerámica de cocción oxidante, pasta compacta, con buena resistencia a la fractura e inclusiones de fracción homogénea y muy fina. El tratamiento de superficie se presenta en ambos lados (interior y exterior) con un alisado fino y apariencia de pulido. La decoración se representó en forma de guarda la cual contiene líneas quebradizas que delimitan campos más pequeños. Éstas se realizaron mediante trazos gruesos que se efectuaron por incisión. Las mismas delimitan espacios rellenos de trazos lineales más finos dispuestos paralelamente en sentido vertical (Figura 7). Tanto el tamaño del fragmento $(6,3 \times 4,8 \mathrm{~cm})$ como el espesor de sus paredes $(0,36 \mathrm{~cm})$ y sus proporciones decorativas nos sugieren que el mismo formó parte de un contenedor de pequeño a mediano tamaño, como puco o vaso.

Sobre las huellas de uso observadas en este fragmento, relevamos una perforación localizada en un extremo del mismo, lo cual sugiere un rastro de reparación de la pieza.

Teniendo en cuenta las características mencionadas, consideramos que el Fragmento 1 es semejante al estilo cerámico Ciénaga y que forma parte de alguno de los recipientes más representados en los contextos funerarios del área valliserrana: urnas, vasos, pucos o escudillas (Balesta y Zagorodny 2002). Además, es importante señalar que en dichos espacios se recuperaron numerosos contenedores cerámicos con agujeros de reparación (Balesta y Zagorodny 2002). En relación a esto, las investigadoras conjeturan que la alfarería podría haberse usado en diferentes contextos previo a su incorporación a los entierros; pues una vez fracturadas y reparadas las piezas, podrían continuar utilizándose como contenedores de sólidos de grano grueso. Para conocer con mayor profundidad aquellos usos que no dejaron huellas identificables a ojo desnudo, sería apropiado realizar análisis de microrrestos, posiblemente depositados en la superficie interna del fragmento. Esto nos posibilitaría observar con mayor claridad si los artefactos cerámicos depositados fueron utilizados, previamente a la inhumación, en otras actividades.

La presencia del estilo cerámico Ciénaga (junto con Saujil) en la micro-región puneña, no representa una novedad dado que esta alfarería es registrada constantemente en todo tipo de contextos, incluso pudiendo apreciarse una incrementada representatividad de esta cerámica valliserrana desde el 1.800 AP en adelante (Olivera 1992).

En relación a las características petrográficas del Fragmento 1 se identificó una pasta compacta la cual posee una matriz arcillosa de color pardo y textura pseudolepidoblástica. La misma contiene finas laminillas de biotita. Sus cavidades se presentan en porcentajes moderados (5-15\%), con formas alargadas y una orientación regular. Entre los antiplásticos se registraron mineraloclastos de cuarzos, feldespatos potásicos, micas, plagioclasas. Asímismo, la pasta contiene materiales volcánicoclásticos como trizas volcánicas, escorias y fragmentos pumíceos. Estas inclusiones se presentan en porcentajes bajos (5-10\%). Son angulares, subangulares y suredondeadas con una orientación regular parcial y poseen granulometría de limo medio a arena fina. Dada la abundante presencia de materiales volcánicos en la micro-región de ANS, es que postulamos la posible procedencia local de esta cerámica pero con representación estilística alóctona.

\section{El rol social de la cerámica en los contextos funerarios}

Respecto a la Pieza 1 registrada como contenedor de inhumación en el contexto funerario de PP9-III, fue posible identificar mediante los estudios estilísticos efectuados, una alfarería con características de manufactura local. Así también observamos en este recipiente la presencia de fracturas significativas junto a evidencias de uso intensivo. A partir de esto nos preguntamos en primera instancia sobre el significado del estado de fragmentación de los 
artefactos que participan en estos espacios particulares.

En el abordaje de esta problemática debemos tener en cuenta que, además de la Pieza 1, se registraron en el contexto funerario de PP9-III más artefactos, de los cuales algunos se presentaron fracturados como, por ejemplo, una pala lítica y una cuenta ${ }^{3}$ de malaquita la cual se fracturó durante su formatización (Cohen 2005). En relación a esto, Cohen propone que los ítems registrados como rotos en tales espacios puedan estar vinculados con una preferencia de esas características para hacerlos partícipes de las prácticas funerarias. Asimismo, creemos que podemos contribuir a esta interpretación con los resultados de los análisis de huellas de uso efectuados sobre la Pieza 1. Éstos nos permitieron identificar rastros de adherencias como hollín y fósiles de micro-carbones, y desgastes como rastros de abrasión por incisión y raspaduras. Estas alteraciones nos dan indicios de un uso intenso y probablemente prolongado del artefacto en actividades relacionadas con el procesamiento de sustancias y exposición al calor, lo cual pudo haber sido la principal causa para su rotura. Así, en condiciones de desgastes pronunciados y con fracturas, se incorporó la pieza al contexto de inhumación para la contención del neonato.

En relación a una posible preferencia de los sujetos por incorporar elementos rotos al contexto de inhumación, desde los estudios efectuados a la Pieza 1 podemos contribuir con lo siguiente:

La pieza evidencia diversas huellas de uso que sugieren un uso intensivo, lo cual pudo provocar la erosión y fractura del artefacto, imposibilitando su continuidad funcional en actividades relacionadas con la contención de líquidos y semi-líquidos y/o el procesamiento de sustancias. Tal biografía del objeto (sensu Gosden y Marshall 1999) pudo pesar en una valoración positiva del mismo, incentivando su selección para la participación de este artefacto en condiciones de roturas o desgaste en los contextos funerarios. De esta manera, interpretamos que el rol que tuvo la participación de objetos como la Pieza 1 en los contextos de inhumación, tiene que ver con la cualidad del objeto como portavoz de una historia probablemente familiar o relacionada con quién o quiénes interactuaron directamente con el contenedor en actividades preinhumación.

En relación a la Pieza 2 (del Contexto Funerario A de PP13-I), consideramos que esta vasija reflejaría las interacciones existentes entre la población puneña y algunos grupos sociales de las selvas occidentales meridionales del NOA. Los elementos que evidenciarían tal acercamiento entre estas poblaciones son: el estilo cerámico Candelaria representado en el recipiente depositado en ANS, la práctica de inhumación asociada (diferente de las

\footnotetext{
${ }^{3}$ La cuenta de $4 \mathrm{~mm} \times 2 \mathrm{~mm}$ conserva un borde con arista y otro redondeado.
}

frecuentemente registradas en la microregión puneña) y los restos vegetales alóctonos identificados (como las especies Bromeliaceae y Arecaceae). Teniendo en cuenta la presencia recurrente de evidencias alóctonas en ANS y en diversos contextos de hasta 10.000 años de antigüedad (incluyendo los funerarios), Aschero (2007) menciona que los eventos de interacción entre la población local y la extra-puneña no se limitaron al intercambio y/o circulación de bienes económicos, sino que esta dinámica también conllevó la creación de relaciones de parentesco.

Por otra parte, es posible que la incorporación de la Pieza 2 al contexto funerario esté mediada por el acondicionamiento del contenedor mediante la realización de los agujeros como práctica de "matado". Este atributo es interpretado por algunos autores como el resultado de acciones cuya finalidad o rol es acompañar a los difuntos (Román Marambio y Cantarutti Rebolledo 1998).

En la Pieza 3 identificamos la confluencia de prácticas vinculadas al uso de recursos locales para representar estilos cerámicos definidos en un área extra-puneña. Por ello, consideramos que tal coexistencia sugiere que las relaciones entre poblaciones que interactúan posiblemente por lazos de parentesco (entre otros motivos), pueden estar siendo expresadas mediante la materialización y presencia de la Pieza 3 en el contexto de inhumación. Así también, teniendo en cuenta la asociación en el contexto funerario entre la Pieza 3 y la Pieza 2 con características estilísticas Candelaria, y los ecofactos confeccionados en fibras vegetales de especies foráneas a la puna, es posible pensar al Contexto de Funerario A de PP13-I, en su totalidad, como una forma de expresión material de lazos de parentesco entre la población puneña y grupos particulares de las tierras bajas orientales.

Por su parte, el Fragmento 1 (asociado a un depósito funerario de características locales en Contexto Funerario B de PP13-I) presenta semejanzas estilísticas a la cerámica Ciénaga, por lo cual, inferimos que la presencia de estos objetos en ANS representa una acercamiento simbólico entre los grupos sociales de valles y los puneños. Dicho acercamiento, estaría dando cuenta de la interacción existente y persistente en el tiempo entre las poblaciones, la cual tendría una profundidad temporal que se remite a los inicios de la era cristiana (Olivera 1992).

La interacción entre puneños y extra-puneños la vemos además en el Fragmento 1 donde la decoración vallista se presenta en una cerámica manufacturada con materias primas, que en esta etapa de análisis, se determinaron como coherentes con la geología regional. En relación a esto, Aschero (2007) propone que tales prácticas pudieron tener lugar durante la presencia de personas con conocimientos diferentes a los alfareros de la puna. Estos conocimientos pudieron compartirse o adquirirse 
mediante interacción y/o porque las manufacturas fueron realizadas por personas provenientes de otras regiones, quizás mujeres de otras puntos geográficos elegidas como cónyuges, que traen cerámica de su lugar de origen o confeccionan vasijas con recursos locales pero mediante modos de hacer por ellas conocidos (Aschero 2007). Para dilucidar los modos de hacer, locales o alóctonos, debemos avanzar en mayor profundidad sobre el estudio de las secuencias productivas de la cerámica.

\section{Consideraciones finales}

El estudio estilístico y de huellas de uso efectuado sobre la cerámica, nos permitió abordar aquellas características que pudieron primar en la selección de los objetos para incorporarlos a los contextos funerarios y acercarnos a conocer el rol social que cumplió cada artefacto en esos espacios. En relación a la Pieza 1 (PP9- III) destacamos sus atributos de manufactura local y la presencia de rastros de desgaste que sugieren un uso intensivo y posiblemente prolongado en el tiempo. A partir de esto, interpretamos que la incorporación de la Pieza 1 al contexto funerario puede responder a las características estilísticas locales del artefacto como así también a su trayectoria de vida, relacionada con múltiples usos, en pos de representar una historia probablemente familiar o relacionada con quién o quiénes interactuaron directamente con el contenedor en actividades pre-inhumación.

Por otra parte, en relación a la presencia de estilos cerámicos alóctonos en contextos funerarios de ANS como la Pieza 2, Pieza 3 y Fragmento 1 (PP13-I), pensamos que estos materializan los vínculos entre personas de diferentes regiones, muy posiblemente emparentados considerando además que éstos fueron registrados en asociación a inhumaciones de niños. Desde esta perspectiva, adherimos a la propuesta de Aschero (2007), quien considera que la profundidad temporal de las relaciones extra-regionales y en especial entre ANS y los valles pudo conducir a un tipo de filiación entre las poblaciones mencionadas que exceden las interacciones e intercambios a nivel económico. Por su parte, esos vínculos estarían asegurando y dando legitimidad a las relaciones creadas mediante la construcción de filiaciones parentales entre los integrantes de las diferentes regiones.

Finalmente, podemos decir que la cerámica, desde sus particularidades estilísticas y su relación con los contextos de hallazgo, constituye un elemento significativo para aportar sobre la dinámica social de las poblaciones. Asimismo, mediante un análisis contextual e integrador, consideramos que es posible aportar interpretaciones más profunda sobre la participación de los objetos cerámicos en diversos tipos de actividades delineando sus biografías y las pautas que primaron en la selección de tales objetos para tales contextos. Sin embargo, las ideas expuestas, lejos de ser concluyentes pretenden incentivar la discusión sobre las diferentes estrategias posibles de abordar para el estudio de la cerámica y sus múltiples propiedades constitutivas.

San Miguel de Tucumán, 18 de Julio de 2016.

\section{Agradecimientos.}

El presente trabajo se desprende de mi tesis de grado, la cual se desarrolló dentro del marco de una beca "Estímulo a las Vocaciones Científicas" otorgada por el Consejo Interuniversitario Nacional. Además, la investigación también se llevó a cabo dentro del proyecto PIUNT60120120100442TU, titulado "Abriendo espacios... perspectivas sobre modos de vida en la larga duración para la Puna Argentina: Antofagasta de la Sierra y El Aguilar como casos", dirigido por el Lic. Carlos Alberto Aschero a quien agradezco su apoyo en la realización de este trabajo.

Además, quiero expresar mi agradecimiento al Arql. Mariano Corbalán y la Dra. Lorena Cohen quienes se encargaron dirigirme en el desarrollo de esta investigación con mucha dedicación; al Dr. A. Martel y la Dra. S.M.L. López Campeny por facilitarme el material de estudio y la información por ellos generada previamente; a la Dra. V. Puente quien también tuvo la cortesía de facilitarme información inédita; a la Dra. Beatriz Cremonte y Dra. Clarisa Otero quienes amablemente leyeron este artículo y expresaron valiosos aportes. Así también, agradezco a Julieta Zapatiel y Cecilia Haros por su colaboración en el análisis de microfósiles. Finalmente gracias al Arql. Matías Lepori por su disposición de siempre para colaborar en las traducciones castellano-inglés.

\section{Bibliografía}

Ambrosetti, J. B.1906. Exploraciones arqueológicas en la Pampa Grande (provincia de Salta). Imprenta Didot de Félix Lajouane y Co. Buenos Aires.

Arnold, D. 2005. Linking Society with the Compositional Analyses of Pottery: A Model from Comparative Ethnography. En: A. Livingstone Smith, D. Bosquet and R. Martineau eds. En: Pottery Manufacturing Processes: Reconstruction and Interpretation.

Aschero, C.A. 2007. Comentario. Mesa 1: Interacciones surandinas. Aspectos económicos, políticos e ideológicos. En Sociedades Precolombinas Surandinas: Temporalidad, interacción y dinámica cultural del NOA en el ámbito de Ios Andes Centro-Sur, editado por V. Williams, B. Ventura, A. Callegari y H. Yacobaccio. Pp 99-108, Buenos Aires.

Aschero, C. A. y S. Hocsman. 2011. Arqueología de las ocupaciones cazadoras-recolectoras de fines del holoceno medio de Antofagasta de la Sierra (Puna meridional argentina). En Chungara, Revista de Antropología Chilena. Vol 43 (Número especial 1): 393-411. 
Aschero, C.A., R. Zurita, M.G. Colaneri y A. Toselli. 2002. El bebé de la Peña. Actas XIII Congreso Nacional de Arqueología Argentina, Tomo 2: 329-336. Universidad Nacional de Córdoba.

Babot, M. del P., L. González Baroni, S. Urquiza, M.G. Aguirre, M.G. Colaneri, S. Hocsman y C. Haros. 2009. Dinámicas de formación y transformación de un entierro en el desierto puneño (Antofagasta de la Sierra, Puna Meridional Argentina). En Intersecciones en Antropología. Vol 10:183-201.

Balesta, B., N. Zarogodny. 2002. La restauración alfarera en la funebria arqueológica. Observación y estudios experimentales sobre la Colección Muñíz Barreto. En Bull. Inst. Fr. Études andines 31(2): 373-395.

Balfet, H., M. F. Fauvet-Berthelot, y S. Monzon. 1992. Normas para la descripción de vasijas cerámicas. En Centro de Estudios Mexicanos y Centroamericanos, México.

Berberián, E. E. 1969. Enterratorios de adultos en urnas en el Área Valliserrana del Noroeste Argentino. En Publicaciones del Instituto de Antropología 29: 3-71.

Cohen, M. L. 2005. “Entre guano y arena. Ocupaciones recurrentes: Un caso de estudio en el sitio Punta de la Peña 9-III. Antofagasta de la Sierra, Catamarca". Facultad de Ciencias Naturales e IML. Universidad Nacional de Tucumán, Argentina. 251 Pág. Tesis de Licenciatura. Biblioteca del IAM.

Cohen, M.L. 2010. “Prácticas sociales, estratégias de visibilidad y construcción de La cartografia social durante el lapso Ca. 1000-1500 AD en Antofagasta de la Sierra, Catamarca. Perspectivas desde El sitio Peñas Coloradas 3 cumbre". Facultad de Filosofía y Letras. UBA. Argentina. 490 Pág. Tesis Doctoral.

Del Bel, E., S. Ibañez, J., Medina Chueca, G. Ortiz, G. Rodríguez y S. Curletto. 2006. Informe final inédito de la Materia de Especialidad Paleopatologías Humanas. Carrera de Arqueología, Facultad de Ciencias Naturales e I.M.L, Universidad Nacional de Tucumán.

González, O. E. 1983. Geología, alteración hidrotermal y edad del cerro Tebenquicho, Depto. Antofagasta de la Sierra- Catamarca. En Revista Asociación Geológica Argentina XXXVIII (1): 49-59. Buenos Aires.

González Baroni, L.G. 2013. "Contextos funerarios y vida cotidiana en Antofagasta de la Sierra, Catamarca. Un enfoque desde la Bioantropología, el emplazamiento y la dinámica de los entierros (ca. 1500-1000 AP)". Facultad de Ciencias Naturales e I.M.L. Universidad Nacional de Tucumán. Argentina. 280 Pág. Tesis de Grado. Biblioteca del IAM.
Gosden, C. y Marshall Y. 1999. The cultural biography of objects. En World Archaeology, Vol. 31 (N²): 169-178.

Haros, M. C. 2014. Informe inédito de microfósiles. Instituto de Arqueología y Museo. Universidad Nacional de Tucumán.

Heredia, O. 1974. Investigaciones arqueológicas en el sector meridional de las selvas occidentales. En Revista del Instituto de Antropologia, Vol 4: 73-132.

Hodder, I. 1990. Style as Historical Quality. En M. Conkey. y C.Hastorf (Eds.). The Uses of Style in Arqueology. Pp 44-51. Cambridge, Cambridge University Press.

Krapovickas, P. 1955. El yacimiento Tebenquiche (Puna de Atacama). Publicaciones del Instituto de Arqueología III, Universidad de Buenos Aires, Buenos Aires.

López Campeny S. M. L. 2009. “Asentamiento, Redes sociales, Memoria e Identidad. Primer milenio de la era. Antofagasta de la Sierra, Catamarca. Facultad de Ciencias Naturales y Museo, Universidad Nacional de La Plata, Argentina, 661 Pág. Tesis Doctoral inédita.

López Campeny S. M. L. 2012.¿De Valles o de Puna? Discutiendo interacción a partir de la caracterización composicional de conjuntos cerámicos. El caso de Antofagasta de la Sierra, Puna sur argentina. En Estudios Atacameños. Vol 43: 139-166.

López Campeny, S. M. L., A. S. Romano, M. F. Rodríguez, A. R. Martel y M. H. Corbalán. 2014. De aquí y de allá: análisis integral de un contexto funerario. Vínculos e interacciones sociales entre Puna meridional y Tierras Bajas orientales. En Intersecciones en Antropología. Vol 15: 201-218.

Mapa Geológico de la Provincia de Catamarca. 1995. Secretaría de Minería. Dirección Nacional del Servicio Geológico. Escala 1: 500.000.

Mapa Geológico de la Provincia de Jujuy. 1996. Secretaría de Industria, Comercio y Minería. Dirección Nacional del Servicio Geológico. Instituto de Geología y Minería . Universidad Nacional de Jujuy. Escala 1:500.000.

Mapa Geológico de la Provincia de Salta. 1998. Secretaría de Industria, Comercio y Minería. Servicio Geológico Minero Argentino. Instituto de Geología y Recursos Minerales. Escala 1: 500.000 .

Mapa Geológico de la Provincia de Tucumán. 1994. Secretaría de Minería. Dirección Nacional del Servicio Geológico. Escala 1: 500.000.

Martel, A. R. 2005. Resumen de las actividades llevadas a cabo en el sitio Punta de la Peña 13 (PP13). Campañas 
Abril 2003 y Mayo 2004. Investigaciones arqueológicas en el área del curso medio del Río Las Pitas y curso alto del Río Miriguaca, Período 2003-2004. C. A. Aschero (Coord.). Informe Inédito elevado a la Dirección de Antropología de la Provincia de Catamarca.

Martínez, J.G. y C.A. Aschero. 2005. Investigaciones en el sitio Peñas de las Trampas 1.1: entre megafauna y contextos funerarios (Antofagasta de la Sierra, Catamarca). En VII Jornadas de Comunicaciones. Serie Monográfica y Didáctica No 45:25. Fac. de Ciencias Naturales e Instituto Miguel Lillo. UNT.

Martínez, J. G., N.M. Mondini, E. Pintar, M. del C. Reigadas. 2010. Cazadores-recolectores tempranos de la Puna Meridional Argentina: avances en su estudio en Antofagasta de la Sierra (Pleistoceno Final-Holoceno Temprano/Medio). En Actas del XVII Congreso Nacional de Arqueología Argentina. Tomo 4 (32): 1691-1696. Mendoza.

Olivera, D. P. 1992. "Tecnología y estrategias de adaptación en el Formativo (Agro-alfarero Temprano) de la Puna Meridional Argentina. Un caso de estudio: Antofagasta de la Sierra (Pcia. De Catamarca, R.A.)". Facultad de Ciencias Naturales, Universidad Nacional de la Plata, Argentina, $412 \mathrm{Pp}$. Tesis Doctoral inédita para optar al grado académico de Doctor en Cs. Naturales. Instituto Nacional de Antropología y Pensamiento Latinoamericano. Centro de Documentación e Información.

Olivera, D. P., P. Escola, A.M. Elías, S. Pérez, P. Tchilinguirian, P. Salminci, M. Pérez, L.G. Grana, J. Grant Lett-Brown, A. Vidal, V. Killian Galván, P. Miranda. 2006. El Formativo en la Puna Meridional: de la opción productiva a las sociedades agropastoriles plenas. En Instituto Superior de Estudios Sociales Link: http//:,www.ises. org.ar/arqueología//pdf/201120143937175039.pdf. (14/07/2016).
Orton, C., P. Tyres, A. Vince, R. Barceló y J. A. Barceló. 1997. La cerámica en Arqueología. Ed. Crítica. Barcelona.

Palma, M.A; P.D. Parica y V.A. Ramos. 1986. El granito Archibarca: Su edad y significado tectónico, Provincia de Catamarca. En Revista Asociación Geológica Argentina XLI (3-4): 414-419. Buenos Aires.

Primera Convención Nacional de Antropología. 1966. Facultad de Filosofía y Humanidades, Instituto de Antropología, Universidad Nacional de Córdoba. Publicación $\mathrm{N}^{\circ}$ 1: 24-48.

Puente, V. 2015. Relaciones de interacción entre Antofagasta de la Sierra y el Valle del Bolsón (Catamarca, Argentina). Primeros aportes desde la alfarería ca. 9001600 D.C. En Chungara, Revista de Antropología Chilena. Vol 47 (N³): 369.

Rice, P. 1989. Ceramic Diversity, Production and Use. (Eds. Leonard, R. D. and Jones, G. T). Quantifying Diversity and Archaeology, 109-117. Cambridge, University Press. Cambridge.

Román Marambio, G. y G. Cantarutti Rebolledo. 1998. Hallazgo de perforaciones basales en la alfarería Diaguita: Una aproximación desde la restauración y la investigación arqueológica de colecciones. En Conserva N²: 81-100. Universidad de Chile.

Skibo, J. M. 1992. Pottery Function. A Use-Alteration Perspecive. Plenum Press, New York.

Wynveldt, F. 2006. "Funcionalidad y cronología en un Sitio del Período de Desarrollos Regionales (Loma de los Antiguos, Dpto. Belén, Catamarca)". Facultad de Ciencias Naturales y Museo. Universidad Nacional de la Plata. Argentina, 249 Pág. Tesis para optar por el grado de Doctor en Cs. Natrales inédita. Fac. de Cs. Nat. y Museo. 